\title{
Rotational Hysteresis of Exchange-Spring Magnets
}

\author{
J. S. Jiang, S. D. Bader, H. Kaper, G. K. Leaf \\ Argonne National Laboratory, Argonne, IL 60439 \\ R. D. Shull, A. J. Shapiro, V. S. Gornakov, V. I. Nikitenko, \\ National Institute of Standards and Technology, Gaithersburg, MD 20899 \\ C. L. Platt, A. E. Berkowitz \\ University of California, San Diego, La Jolla, CA 92093 \\ S. David, and Eric E. Fullerton \\ IBM Almaden Research Center, 650 Harry Road, San Jose, CA 95120
}

\begin{abstract}
We highlight our experimental studies and micromagnetic simulations of the rotational hysteresis in exchange-spring magnets. Magneto-optical imaging and torque magnetometry measurements for $\mathrm{SmCo} / \mathrm{Fe}$ exchange-spring films with uniaxial in-plane anisotropy show that the magnetization rotation created in the magnetically soft Fe layer by a rotating magnetic field is hysteretic. The rotational hysteresis is due to the reversal of the chirality of the spin spiral structure. Micromagnetic simulations reveal two reversal modes of the chirality, one at low fields due to an in-plane untwisting of the spiral, and the other, at high fields, due to an out-of-plane fanning of the spiral.
\end{abstract}


One of the fundamental issues in magnetism is the magnetization reversal process. In particular, the magnetization reversal properties of exchange-coupled hard/soft magnetic systems are of great current interest. These systems, also known as exchangesprings [1], hold the key to unlocking the potential for technological applications[2,3,4], as well as to elucidating the mechanism for spin transport through magnetic domain walls $[5,6]$.

The simplest realization of the exchange-spring structure is a thin film bilayer consisting of a hard magnetic layer and a soft magnetic layer coupled at the interface through the exchange interaction. [7] Modern thin-film deposition techniques allow for nanometer-scale control of the layer thickness and for tailoring the microstructure, crystal orientation and magnetic anisotropies in the plane of the film through epitaxy. Using bilayer structures of $\mathrm{Sm}-\mathrm{Co} / \mathrm{Fe}$, where the $b$-axis oriented hexagonal $\mathrm{Sm}$-Co layer is grown epitaxially to have a uniaxial in-plane anisotropy, we have studied in detail the magnetization reversal process in exchange-spring magnets by examining the magnetic hysteresis loop measured with the applied magnetic field fixed along the easy axis direction. [8] At reverse fields larger than the exchange field $\mathrm{H}_{\mathrm{ex}}$, the magnetic reversal proceeds via a twisting of the magnetization in the soft layer: the spins that are close to the interface are pinned by the hard layer, while those further away rotate to follow the field. The angle of rotation increases with increasing distance from the hard layer, resulting in a spiral spin structure similar to that in a Bloch domain wall. This process is reversible as the soft spins rotate back into alignment with the hard layer when the applied field is removed. 
The spiral spin structure nucleated during reversal can be either left-handed or right-handed, and both configurations are energetically equivalent when the reverse field is applied precisely along the easy axis of the hard magnetic layer. In real experimental systems, such degeneracy is lifted due to slight misalignment between the easy axis and the applied field [9], or to the microstructural details of the hard layer $[10,11]$. Investigating how the magnetic configuration switches among the opposite chiralities can reveal the energetic competitions, and provide insights to the effect of microstructure on the magnetization reversal process.

This paper highlights our studies of the formation of the spiral spin structure in $\mathrm{Sm}-\mathrm{Co} / \mathrm{Fe}$ exchange-spring bilayer structures in rotating magnetic fields using a magneto-optical imaging technique [12] and torque magnetometry [13]. The magnetization rotation created in the magnetically soft $\mathrm{Fe}$ layer by a rotating magnetic field is hysteretic, due to the reversal of the chirality of the spin spiral structure. Micromagnetic simulations reveal two reversal modes of the chirality, one at low fields due to an in-plane untwisting of the spiral, and the other, at high fields, due to an out-ofplane fanning of the spiral.[13,14]

The exchange-spring films were prepared by dc magnetron sputtering onto a $\mathrm{Cr}$ buffered single-crystalline $\mathrm{MgO}(100)$ or (110) substrates. The Sm-Co layer thickness ranges from 200 to $350 \AA$. The epitaxial growth of the Sm-Co layer gives rise to an inplane, four-fold magnetic anisotropy in the case of $\mathrm{MgO}(100)$ substrates, and an in-plane uniaxial magnetic anisotropy in the case of $\mathrm{MgO}(110)$ substrates. The Sm-Co layers have bulk-like anisotropy fields $(\sim 250 \mathrm{kOe})$ and high coercivity values at room temperature.[15] Once saturated, the Sm-Co layer remains magnetized along an easy 
direction. We can, therefore, make no distinction with respect to the exact nature of its magnetic anisotropy. The magnetically soft Fe layers are exchange coupled to the Sm-Co hard magnet layers, and have thickness values ranging from $50 \AA$ to $500 \AA$.

We have used the magneto-optical indicator film (MOIF) technique to visualize the magnetization rotation process. The MOIF technique [16] is sensitive to stray fields perpendicular to the film plane emanating from magnetic structures, such as from domain walls, Bloch lines, and crystal defects (e.g. dislocations, voids and sample edges). Since the sample magnetization is in-plane, not perpendicular, a small hole $\sim 300 \mu \mathrm{m}$ in diameter was bored through the bilayer sample. Then a thin indicator film of Bi-doped yttrium iron garnet (YIG), having an in-plane magnetization and a large Faraday effect, was placed directly on the sample surface. Around the edge of the hole, the normal component of the leakage field locally causes the magnetization of the indicator film to deviate from its initially in-plane orientation. This deviation induces a Faraday rotation in a polarized light beam, giving rise to black and white optical contrast. The location and intensity of the bright and dark crescents around the hole are determined by the direction and magnitude of the leakage field and provide a means to estimate the average magnetization M. For a Sm-Co(350 $) / \mathrm{Fe}(500 \AA)$ bilayer, the total magnetic moment in the Fe layer is far greater than that in the Sm-Co layer. The magneto-optical (MO) contrast is therefore dominated by the magnetization behavior of the Fe layer.

In Fig. 1 a sequence of MOIF images shows the response of the Fe layer when the applied field is fixed in magnitude but is rotated by angle $\theta$ in the film plane. From the easy magnetization direction (Fig. 1a), as $\theta$ increases, the magnetization smoothly rotates with the field with some phase delay (Fig. 1b). The contrast between the bright 
and dark crescents also decreases with the increasing field angle, indicating an increase in the degree of twisting of the Fe layer spin structure. After the field reaches a critical angle $\theta_{\mathrm{C}}$, the total magnetization begins to rotate in the opposite direction while the field direction continues to change in the original direction (Figs. 1c,d). The subsequent rotation of the total magnetization, after completion of this stage of inverse rotation (Fig. 1e), is again in synchronization with the field. But in this latter case, as one might expect, $\mathrm{M}$ takes the phase lead over $\mathrm{H}$ up to the point where they both coincide with the unidirectional anisotropy axis. Interestingly, a non-uniform MO intensity appears across the sample surface during the inverse rotation of M. This non-uniformity begins to develop in Fig. 1c (or Fig. 1f), and becomes most intense in Fig. 1d (or Fig. 1g) where the effective total magnetization is oriented along the unidirectional anisotropy axis. Finally, the MO signal in the sample again becomes homogeneous (Fig. 3e and 3a). The non-uniform signal is possibly caused by the boundaries between micro-domains where the spin spirals have opposite chirality, due to the dispersion of the easy directions in SmCo layer.[10]

Figure 2 reveals hysteresis in the magnetization rotation. Since the fields available are too small to switch the hard layer, the total magnetization is never reoriented against the unidirectional anisotropy. But we see in Fig. 2 that M jumps to a new equilibrium position, symmetrically oriented with respect to the easy direction, after the field reaches the critical angle $\theta_{\mathrm{C}}$. This angle and the broadness of the transitional region depend on the field magnitude, with a broader transitional region being observed at lower fields. The process for $\mathrm{H}=0.36 \mathrm{kOe}$ is illustrated in Fig. 1. Rotation of the field direction in the opposite sense ( Fig. 1e-h) leads to a magnetization rotation process 
in the opposite direction. This results in a hysteresis in the angular rotation process of the magnetization.

The rotational hysteresis is also seen in torque magnetometry measurements of a Sm-Co(200 A) $/ \mathrm{Fe}(200 \AA)$ bilayer sample. The in-plane measurements were made in a torque magnetometer with applied fields up to $25 \mathrm{kOe}$ and at temperatures from 4 to 350 K. The sample was saturated in the easy axis direction before each measurement, and the torque was measured as the applied field was rotated in the plane of the sample. Shown in Fig. 3(a) are room-temperature torque curves measured for various applied fields. The low-field curve $(\mathrm{H}=0.5 \mathrm{kOe})$ has skewed unidirectional symmetry with little hysteresis, since the soft layer magnetization remains essentially parallel with the hard layer as the field rotates. If the soft layer was rigidly aligned with the hard layer, the torque curve would be sinusoidal, reflecting the Zeeman energy. The skewness indicates some twisting of the Fe layer during rotation. The twisting of the Fe layer is more dramatic in the intermediate field, $\mathrm{H}=4.8 \mathrm{kOe}$. The torque curve remains unidirectional but displays hysteresis about 180 degrees even though the magnetization loop is reversible in this field range. Since the fields are below that required to irreversibly switch the hard layer, the torque curve is dominated by the spring behavior of the soft layer. The hysteresis about $\theta=180^{\circ}$ at intermediate fields results from the irreversible change of the chirality of the twist in the soft layer as the field is rotated past $\theta_{\mathrm{C}}$. When the field is large enough to cause irreversible switching of the hard Sm-Co layer, the torque curves have skewed uniaxial symmetry with hysteretic regions about 90 and 270 degrees. Shown in Fig. 3(b) are the values of $\theta_{\mathrm{C}}$ as a function of applied field measured at both room temperature and $25 \mathrm{~K} . \theta_{\mathrm{C}}$ initially increases, then asymptotically decreases with increasing field and is 
not strongly temperature dependent. Since the coercivity of the Sm-Co layer increases with decreasing temperature, the increased stability of the Sm-Co layer means a stronger twisting of the Fe spins can be supported, therefore the extended ranges of $\mathrm{H}$ and $\theta_{\mathrm{C}}$.

To understand this behavior we compare the experimental results with those from micromagnetic simulations. The exchange-spring bilayer is treated as a chain of spins running normal to the layers. Each spin is the sum of total moments in an atomic layer, and lies in the film plane. The motion of the spins are described by the Landau-LifshitzGilbert (LLG) equation. The local field acting on each spin includes the exchange field from its neighbors, the anisotropy field, the demagnetizing field, and the external applied field H. Figure 4(a) shows the calculated magnetization direction for the $\mathrm{Sm}-\mathrm{Co}(350$ $\AA) / \mathrm{Fe}(500 \AA)$ sample as a function of the field direction at two $\mathrm{H}$ values. There is good qualitative agreement with the MOIF data of Fig. 2. The calculated results possess the same rotational hysteresis loop, and show that the sharp jump in $\alpha$ at $\theta_{\mathrm{C}}$ is due to the irreversible switching of chirality of the spin spiral when one configuration is no longer energetically favorable. The simulation also reproduces the undershoot in angle $\alpha$ just after the chirality reversal for the higher field value. The field dependence of the critical angle shown in Fig. 4(b) also resembles that in Fig. 3(b) from torque measurements.

There is, however, a marked quantitative disagreement in the loop broadness between the calculated and experimental data. The measured loops are much narrower and less sharp than the calculated ones, and $\theta_{\mathrm{C}}$ is far smaller than that calculated at a given field value. The discrepancy arises from the over-simplification of the onedimensional spin-chain model, which assumes that the bilayer films are laterally homogeneous, and overestimates the demagnetization field. In fact, the Sm-Co hard 
layer consists of microscopic grains and regions with different intrinsic properties and varying degrees of stability.[17,18,19] Our recent more quantitative MOIF experiments have further revealed that such dispersion in the directions of the local easy axis leads to laterally non-uniform spin structure and adjacent spin spirals with opposite chiralities.[10] The artificially inflated demagnetization field increases the energy barrier for the chirality reversal, leading to larger calculated $\theta_{\mathrm{C}}$ value than that observed experimentally.

Micromagnetic simulations that take into account the finite lateral dimension of the grains provide further insights into the chirality reversal. The calculations were performed using the LLG micromagnetic code [20] and the film discretized into 16 x 16 cells. The non-monotonic dependence of $\theta_{\mathrm{C}}$ on field reflects two distinct modes for the Fe layer to reverse chirality. For small fields the spins remain in the plane of the films, and at $\theta_{\mathrm{C}}$, the spiral simply unwinds to become parallel with the hard layer and then winds in the opposite direction. (Fig. 5a) The value of $\theta_{\mathrm{C}}$ results from a competition between the exchange energy of the spiral and the Zeeman energy required to unwind the spiral. Thus, $\theta_{\mathrm{C}}$ increases monotonically with increasing applied field as the Zeeman energy increases. For higher fields, we find a second reversal mode, shown schematically in Fig. 5b. During reversal, the spiral structure is maintained and the spins fan out of the plane. This fanning mode avoids the considerable Zeeman energy of unwinding the spiral, and thus $\theta_{\mathrm{C}}$ decreases with increasing applied field.

Further refinement of the numerical simulations is made by taking into account the microstructural characteristics of the Sm-Co layer. Two calculations, one based on the reported tilted epitaxy of the SmCo layers,[15] and the other assuming a reduced 
interfacial SmCo anisotropy, show that the angle decreases for the fanning mode (Fig. 6). The general results of the calculations suggest that variations in the structure tend to lower the energy for the fanning modes, and further demonstrate that local variations and/or defects in the structure play an important role in the magnetization reversal behavior of exchange-spring magnets.

In conclusion we have studied the magnetization reversal behavior of epitaxial exchange-spring $\mathrm{Sm}-\mathrm{Co} / \mathrm{Fe}$ films. We observed hysteretic switching of the soft layer as the field is rotated. The field dependence of the hysteresis can be understood assuming the reversal is controlled by two modes: an unwinding mode at low fields, and a fanning mode at high fields. The quantitative difference between the model calculations and the measured curve suggests that local variations in the Sm-Co anisotropy influence the reversal behavior.

Work at ANL supported by US DOE BES-MS under Contract No. W-31-109ENG-38. 


\section{References}

$1 \quad$ E. F. Kneller and R. Hawig, IEEE Trans. Mag. 27, 3588 (1991).

2 R. Skomski and J. M. D. Coey, Phys. Rev. B 48, 15812 (1993).

$3 \quad$ R. J. Astalos and R. E. Camley, Phys. Rev. B 58, 8646 (1998).

$4 \quad$ T. Ando and Y. Nishihara, IEEE Trans. Mag. 33, 2983 (1997).

$5 \quad$ K. Mibu, T. Nagahama, T. Shinjo, T. Ono, Phys. Rev. B 58, 6442 (1998).

6 S. N. Gordeev, J.-M. L. Beaujour, G. J. Bowden, B. D. Rainford, P. A. J. de Groot, R. C. C. Ward, M. R. Wells, A. G. M. Jansen, Phys. Rev. Lett. 18, 186808 (2001).

$7 \quad$ E. Goto, N. Hayashi, T. Miyashita, K. Nakagawa, J. Appl. Phys. 36 (1965) 2951.

8 E. E. Fullerton, J. S. Jiang, and S. D. Bader, J. Magn. Magn. Mater. 200, 392 (1999).

9 E. E. Fullerton, J. S. Jiang, M. Grimsditch, C. H. Sowers, and S. D. Bader, Phys. Rev. B 58, 12193 (1998).

10 V.S. Gornakov, V.I. Nikitenko, A.J. Shapiro, R.D. Shull, J. S. Jiang, S.D. Bader, J. Magn. Magn. Mater, in press (2002).

11 J. S. Jiang, S. D. Bader, Mater. Sci. Tech. 17, 1491 (2001).

12 R. D. Shull, A. J. Shapiro, V. S. Gornakov, V. I. Nikitenko, J. S. Jiang, H. Kaper, G. Leaf, S. D. Bader, IEEE Trans. Magn. 37, 2576 (2001).

13 C. Platt, A. Berkowitz, S. David, Eric E. Fullerton, J. S. Jiang and S. D. Bader, Appl. Phys. Lett. 79, 3992 (2001).

${ }^{14}$ J. S. Jiang, H. G. Kaper, G. K. Leaf, Discrete and Continuous Dynamical 
Systems, B1, 219 (2001).

Eric E. Fullerton, J. S. Jiang, Christine Rehm, C. H. Sowers, S. D. Bader, J. B. Patel, and X. Z. Wu, Appl. Phys. Lett. 71, 1579 (1997).

L. H. Bennett, R. D. McMichael, L. J. Swartzendruber, S. Hua, D. S. Lashmore, A. J. Shapiro, V. S. Gornakov, L. M. Dedukh, V. I. Nikitenko, Appl. Phys. Lett. 66, 888 (1995).

17 Mohamed Benaissa, Kannan Krishnan, Eric E. Fullerton and J. S. Jiang, IEEE Trans. Mag. 34, 1204 (1998).

18 A. Inomata, J. S. Jiang, C.-Y. You, J. E. Pearson, S. D. Bader, J. Vac. Sci.Technol. A18, 1269 (2000). V. K. Vlasko-Vlasov, U. Welp, J. S. Jiang, D. J. Miller, G. W. Crabtree, S. D. Bader, Phys. Rev. Lett. 86, 4386 (2001).

${ }^{20}$ M. R. Scheinfein, J. Unguris, J. L. Blue, K. J. Coakley, D. T. Pierce, and R. J. Celotta, Phys. Rev. B 43, 3395 (1991) and http://www.dancris.com/ 1lg/. 


\section{Figure Captions}

Figure 1: MOIF images of the perpendicular component of the fringe field near the hole in a $\mathrm{Sm}-\mathrm{Co}(350 \AA) / \mathrm{Fe}(500 \AA)$ sample under a rotating field $\mathrm{H}=0.36 \mathrm{kOe}$ at various angles. The white and black arrows indicate the directions of $\mathrm{H}$ and $\mathrm{M}$, respectively.

Figure 2: Magnetization rotation angle $(\alpha) v s$. the magnetic field direction $(\theta)$ for different field amplitudes measured from the MOIF images.

Figure 3: (a) Room-temperature torque curves for a $\operatorname{SmCo}(200 \AA) / \mathrm{Fe}(200 \AA)$ exchangespring film measured at various applied fields. The curves are measured from 0 to $360^{\circ}$ and back to $0^{\circ}$. Field angles of 0 and $180^{\circ}$ correspond to the easy axis of the SmCo layer. (b) $\theta_{\mathrm{C}}$ values measured at 300 and $25 \mathrm{~K}$.

Figure 4: (a) Simulated magnetization rotation angle $\alpha$ ss. the magnetic field direction $\theta$ for different field amplitudes. (b) Simulated $\theta_{\mathrm{C}}$ values as a function of the magnitude of the applied field.

Figure 5: Schematic representation of the calculated spin structure of the hard and soft layers during reversal through (a) the unwinding mode, and (b) the fanning mode. In the fanning reversal mode, the spins rotate out of the plane of the film. The gray arch at each layer shows the pathway of reversal. 
Figure 6: Simulated $\theta_{\mathrm{C}}$ values for the three model calculations described in the text. Open symbols represent unwinding modes, solid symbols are the fanning mode in the calculations. Square symbols were calculated assuming a uniform structure. Circles assumes that the anisotropy axis of the hard layer is tilted $10^{\circ}$ out of the film plane. Diamonds assume that the interfacial anisotropy of the hard layer is half the bulk value. 

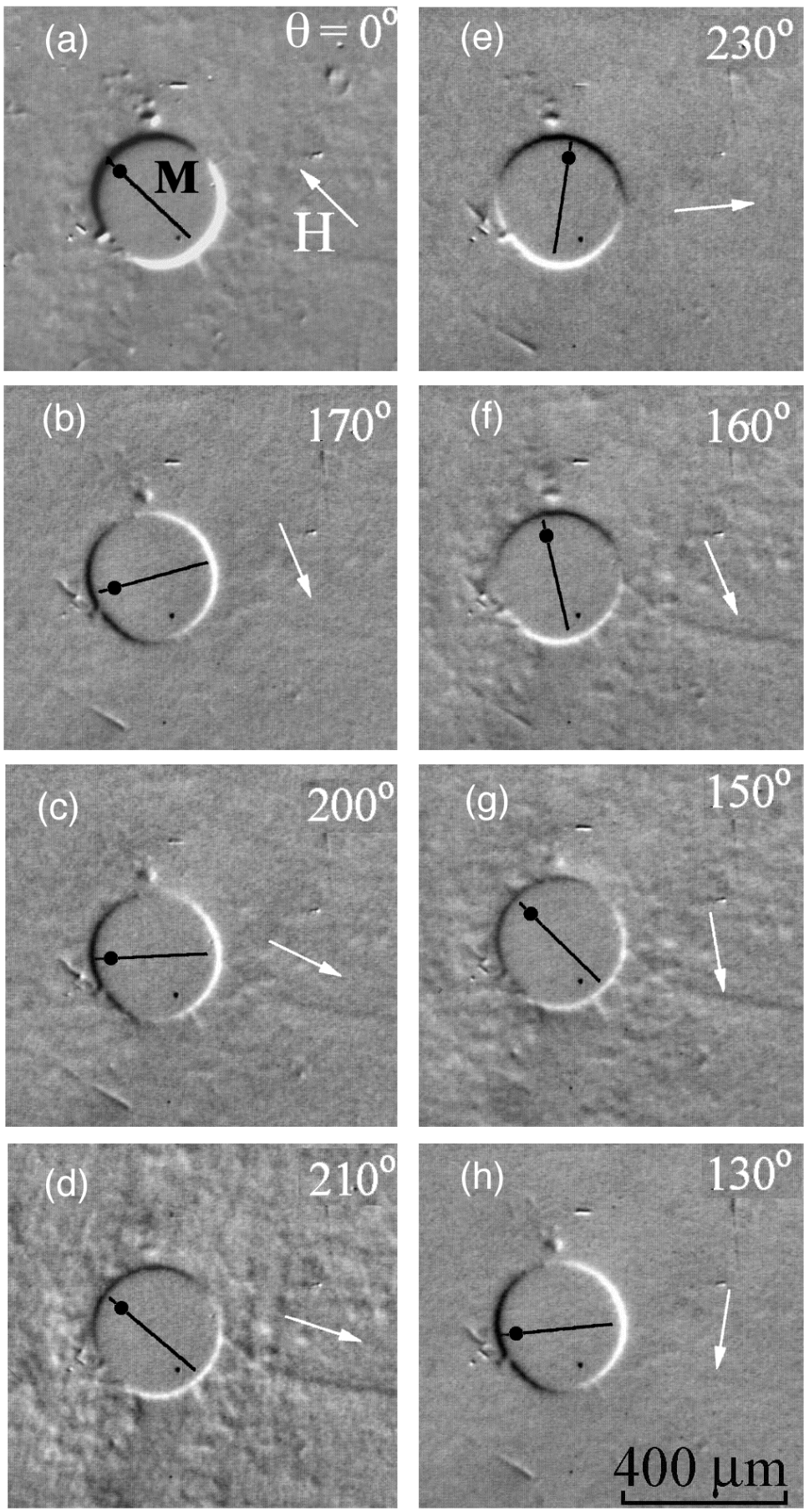

Figure 1 


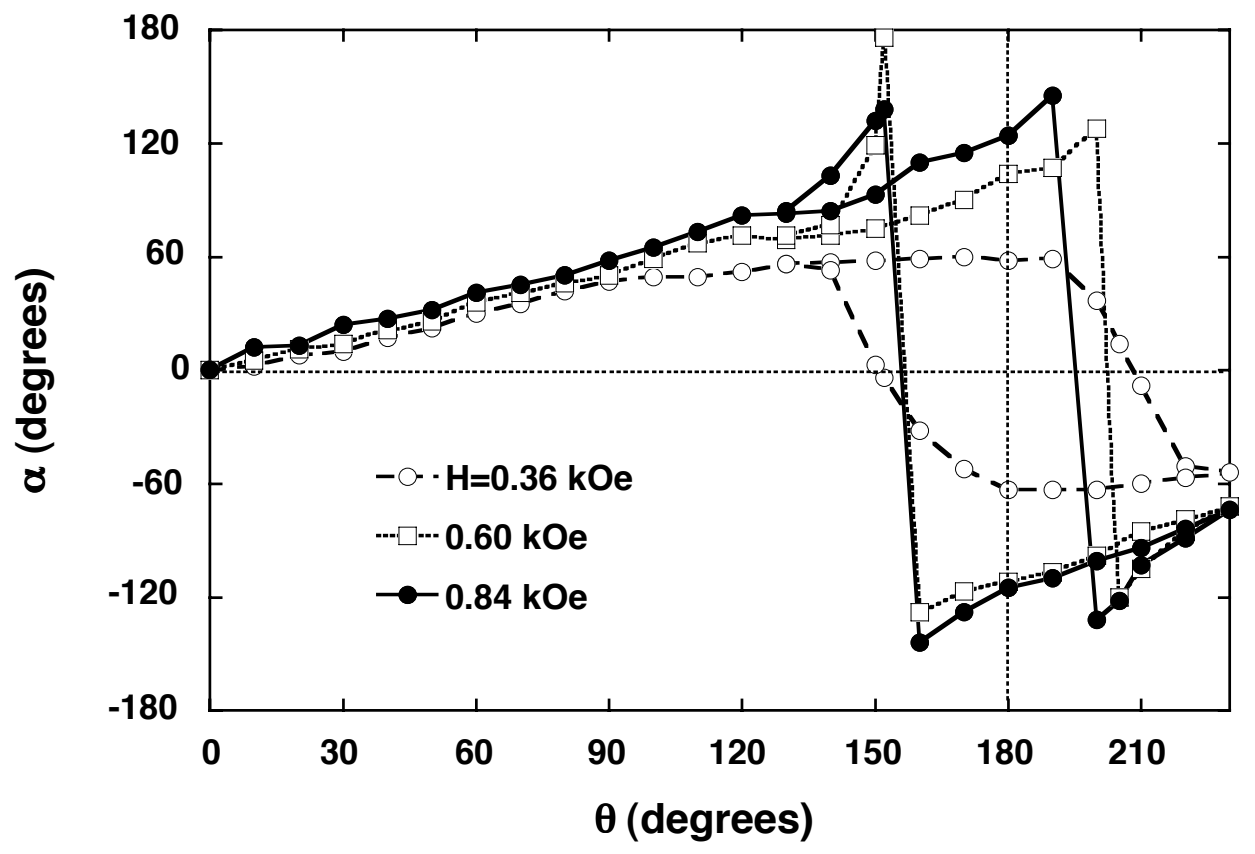

Figure 2 

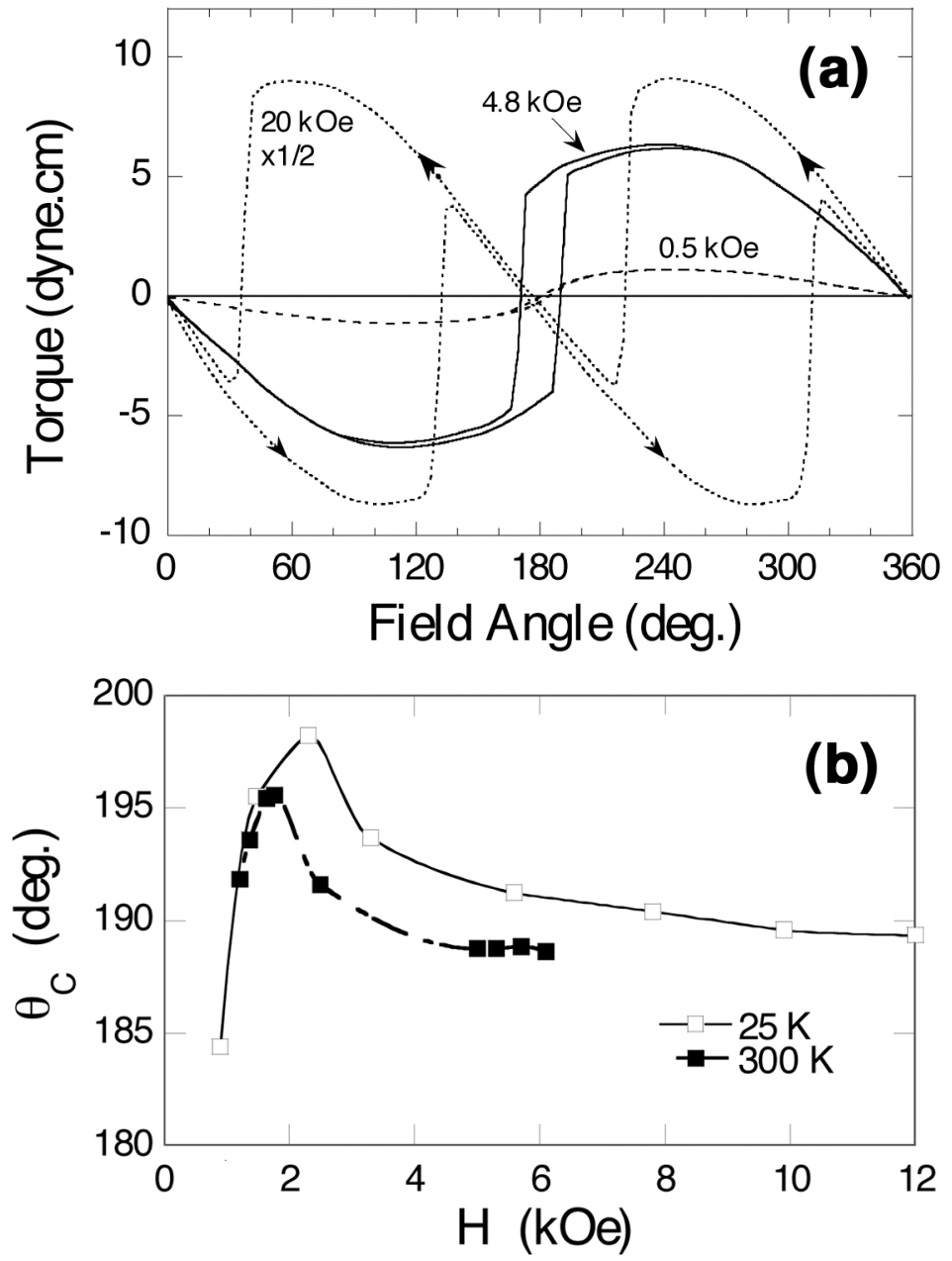

Figure 3 

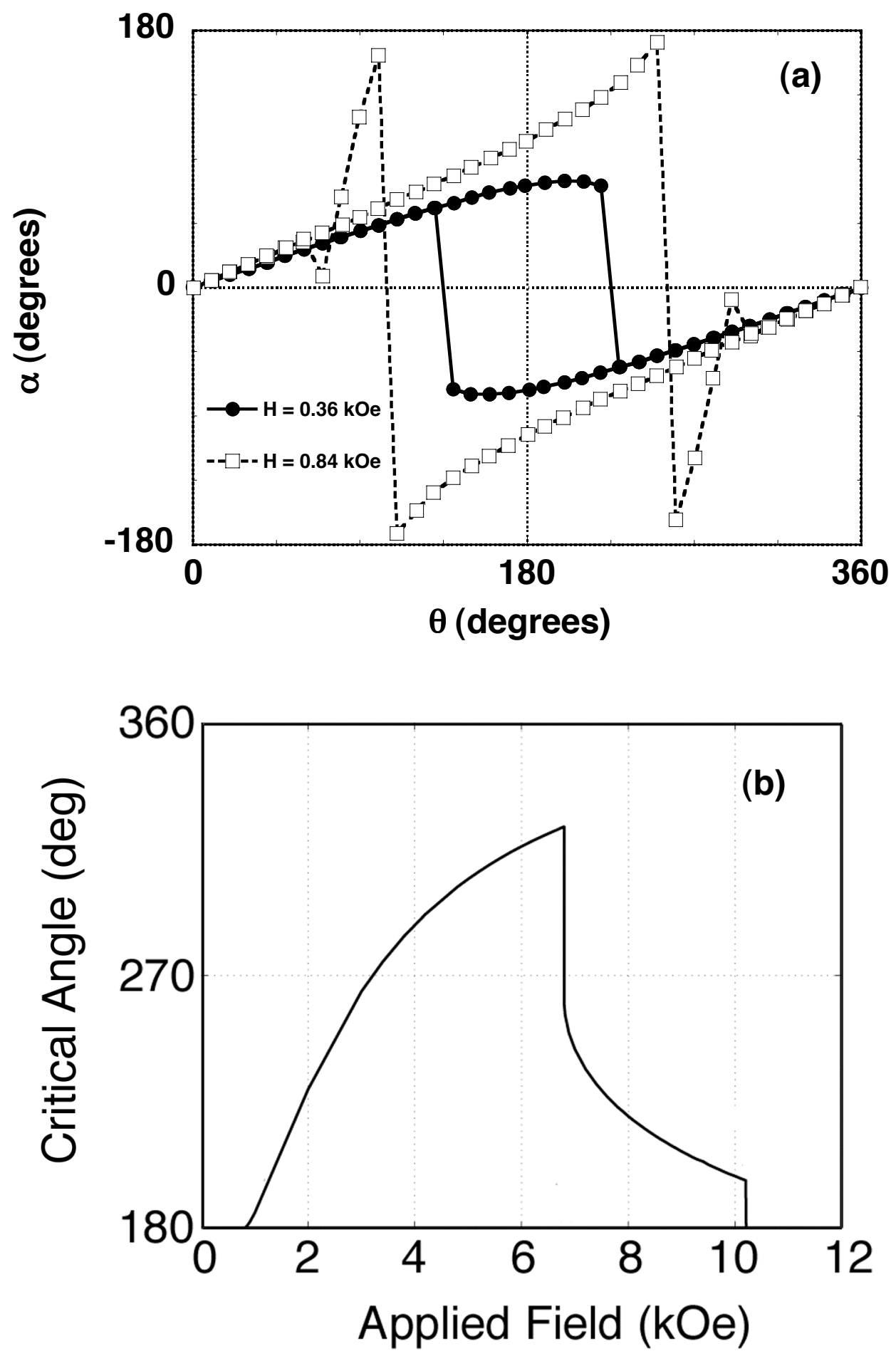

Figure 4 
(a)

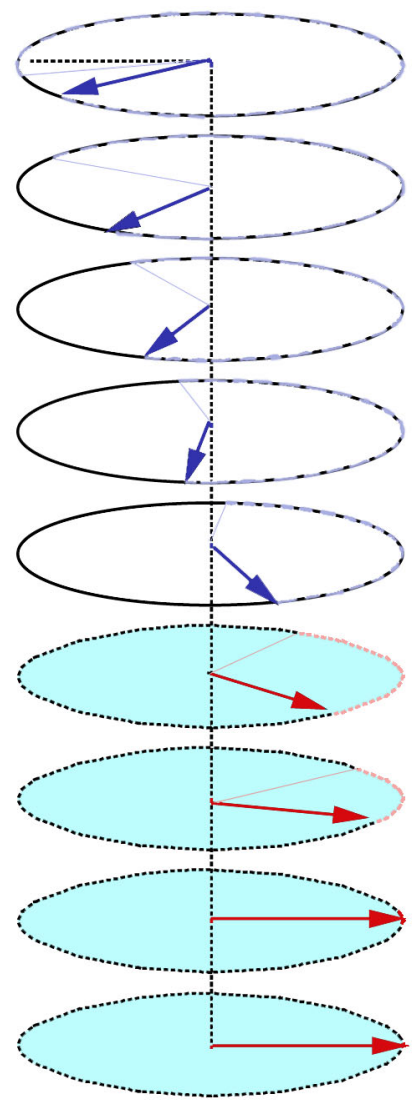

(b)

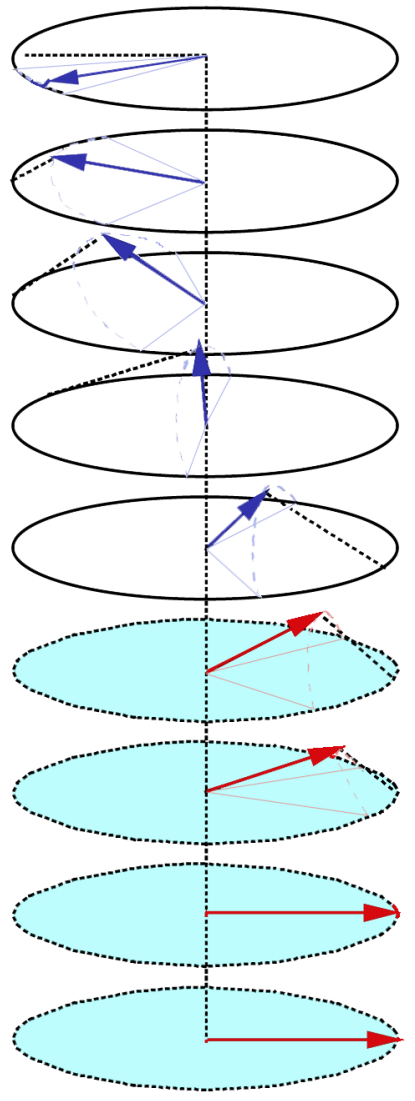

\section{Figure 5}




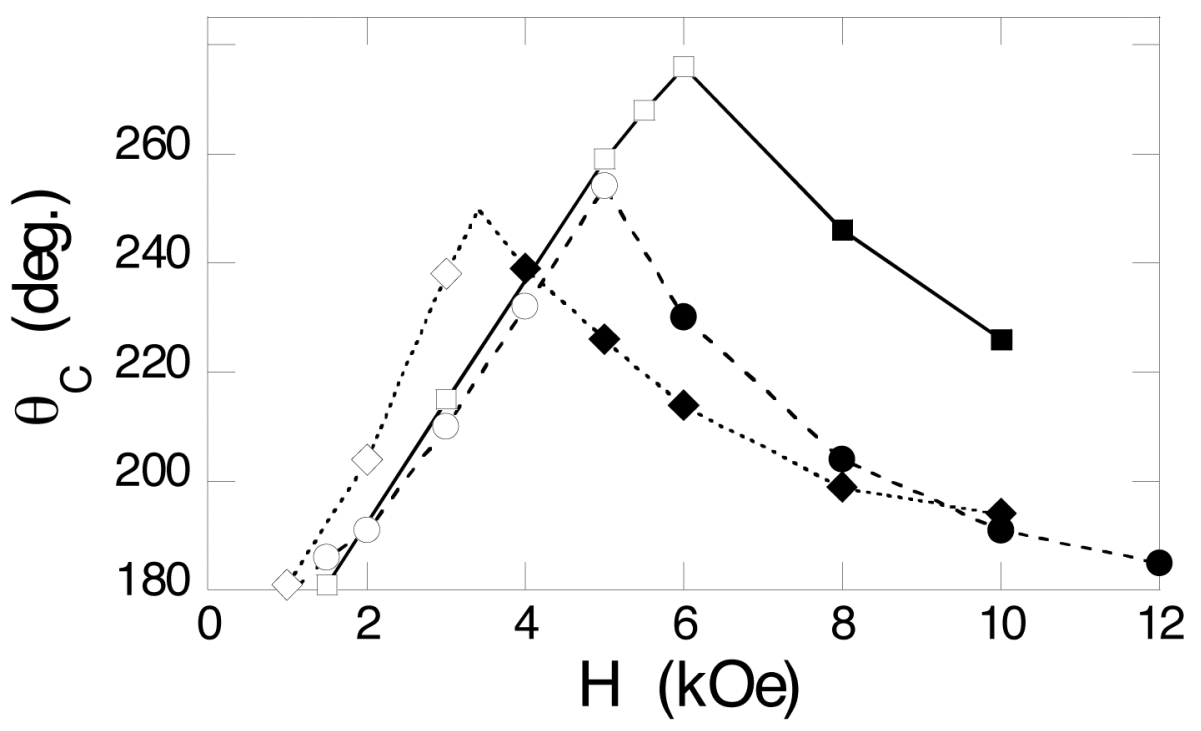

Figure 6 